\title{
A reporter for noninvasively monitoring gene expression and plant transformation
}

\author{
Yubing He(1) ${ }^{1,2}$, Tao Zhang ${ }^{3,4}$, Hui Sun², Huadong Zhan ${ }^{1}$ and Yunde Zhao ${ }^{3}$
}

\begin{abstract}
Reporters have been widely used to visualize gene expression, protein localization, and other cellular activities, but the commonly used reporters require special equipment, expensive chemicals, or invasive treatments. Here, we construct a new reporter $R \cup B Y$ that converts tyrosine to vividly red betalain, which is clearly visible to naked eyes without the need of using special equipment or chemical treatments. We show that RUBY can be used to noninvasively monitor gene expression in plants. Furthermore, we show that $R \cup B Y$ is an effective selection marker for transformation events in both rice and Arabidopsis. The new reporter will be especially useful for monitoring cellular activities in large crop plants such as a fruit tree under field conditions and for observing transformation and gene expression in tissue culture under sterile conditions.
\end{abstract}

\section{Introduction}

Various genetically encodable reporters have been developed to monitor gene expression, protein subcellular localization, protein stability, hormonal signaling, and impacts of environmental signals. The green fluorescent protein (GFP) and its derivatives such as RFP, mCherry, and YFP have many applications as reporters for gene expression or as fusion proteins ${ }^{1,2}$. Although GFP is easy to use, it needs light sources to visualize the fluorescence signals. The $\beta$-glucuronidase (GUS) reporter has been widely used in plants for monitoring gene expression patterns and as a reporter for hormonal signaling ${ }^{3}$. For example, DR5-GUS transgenic lines are commonly used to monitor auxin distribution and auxin signaling ${ }^{4}$. Luciferase is another broadly used reporter in both animals and plants ${ }^{5}$. Both GUS and luciferase require the addition of expensive substrates X-Gluc (5-Bromo-4-

\footnotetext{
Correspondence: Yubing He (yubinghe@njau.edu.cn) or

Yunde Zhao (yundezhao@ucsd.edu)

'State Key Laboratory of Crop Genetics and Germplasm Enhancement, Nanjing Agricultural University, Nanjing 210095, China

${ }^{2}$ National Key Laboratory of Crop Genetic Improvement and National Center of Plant Gene Research (Wuhan), Huazhong Agricultural University, Wuhan

430070, China

Full list of author information is available at the end of the article

These authors contributed equally: Yubing $\mathrm{He}$, Tao Zhang
}

chloro- $1 H$-indol-3-yl $\beta$-D-glucopyranosiduronic acid) and luciferin, respectively. Whereas the traditional reporters have been very useful, they have limitations. Fluorescent proteins are often monitored under a microscope, rendering it less useful in analyzing plants in natural growing fields or analyzing large samples such as a tree. GUSstaining is invasive and often requires sacrifice of the plants. Luciferase can be used noninvasively, but it requires a special camera and spraying the expensive substrate. It is also not very practical to use them in fields. GUS and luciferase may not be optimal for sterile conditions such as tissue culture because addition of substrates increases the chance for contamination of microbes. Therefore, there is a need to develop new reporter systems that can be widely used to monitor cellular activities noninvasively, continuously, and costeffectively. For the past few years, gene editing has been widely used in basic research and crop improvement. A visible marker for transgenes will greatly accelerate the isolation of edited plants that no longer harbor the gene editing machinery ${ }^{6,7}$.

Plants produce many colorful compounds that potentially can serve as reporters. For example, anthocyanins display bright red-blue colors and anthocyanin-producing rice plants have been used to generate interesting patterns 
in rice field. However, synthesis of anthocyanins requires multiple enzymes and varies greatly among different plants $^{8,9}$. It is difficult to use anthocyanin biosynthesis pathways as a universal visible reporter. Betalains are a class of plant natural products derived from the aminoacid tyrosine ${ }^{10,11}$. The bright red color seen in beets, dragon fruit, Swiss chard, and other plants is resulted from accumulation of betalains. Biosynthesis of betalains has been well studied and only needs three enzymatic reactions to convert tyrosine into betalain (Fig. 1a) ${ }^{12}$. Tyrosine is first hydroxylated on the benzene ring, resulting in L-3,4-dihydroxyphenylalanine (L-DOPA) (Fig. 1a). The reaction is catalyzed by the $P 450$ oxygenase CYP76AD1 (Fig. 1a). L-DOPA can be further oxidized into cyclo-DOPA by CYP76AD1 (Fig. 1a). Alternatively, LDOPA is catalyzed by L-DOPA 4,5-dioxygenase (DODA) into betalamic acid, which is subsequently condensed with cyclo-DOPA into betanidin. The condensation reaction does not require an enzyme (Fig. 1a). Finally, a sugar moiety is added to betanidin by a glucosyltransferase to generate the colorful betalain (Fig. 1a). Betalain has a very bright red color, which potentially can serve as a reporter to track gene expression or to visualize transgenic events. Because every cell contains the amino-acid tyrosine, exogenous application of tyrosine to tissues may not be required. We hypothesized that betalain would be a more convenient reporter than the aforementioned reporters. It is visible to naked eyes without any needs for special equipment. It does not require processing samples and it allows continuously monitoring events throughout the life cycle of an organism. Moreover, it is applicable to large plants grown under normal field conditions. Herein, we synthesize an artificial open reading frame named $R U B Y$ that when expressed can produce all of the enzymes required for betalain biosynthesis. We show that $R U B Y$ is a very effective marker for noninvasively selecting transformation events in both rice and Arabidopsis. Moreover, we show that $R U B Y$ can be used to visualize gene expression without any chemical treatments or special equipment, providing useful tools for visualizing gene expression in large plants under natural field growth conditions.

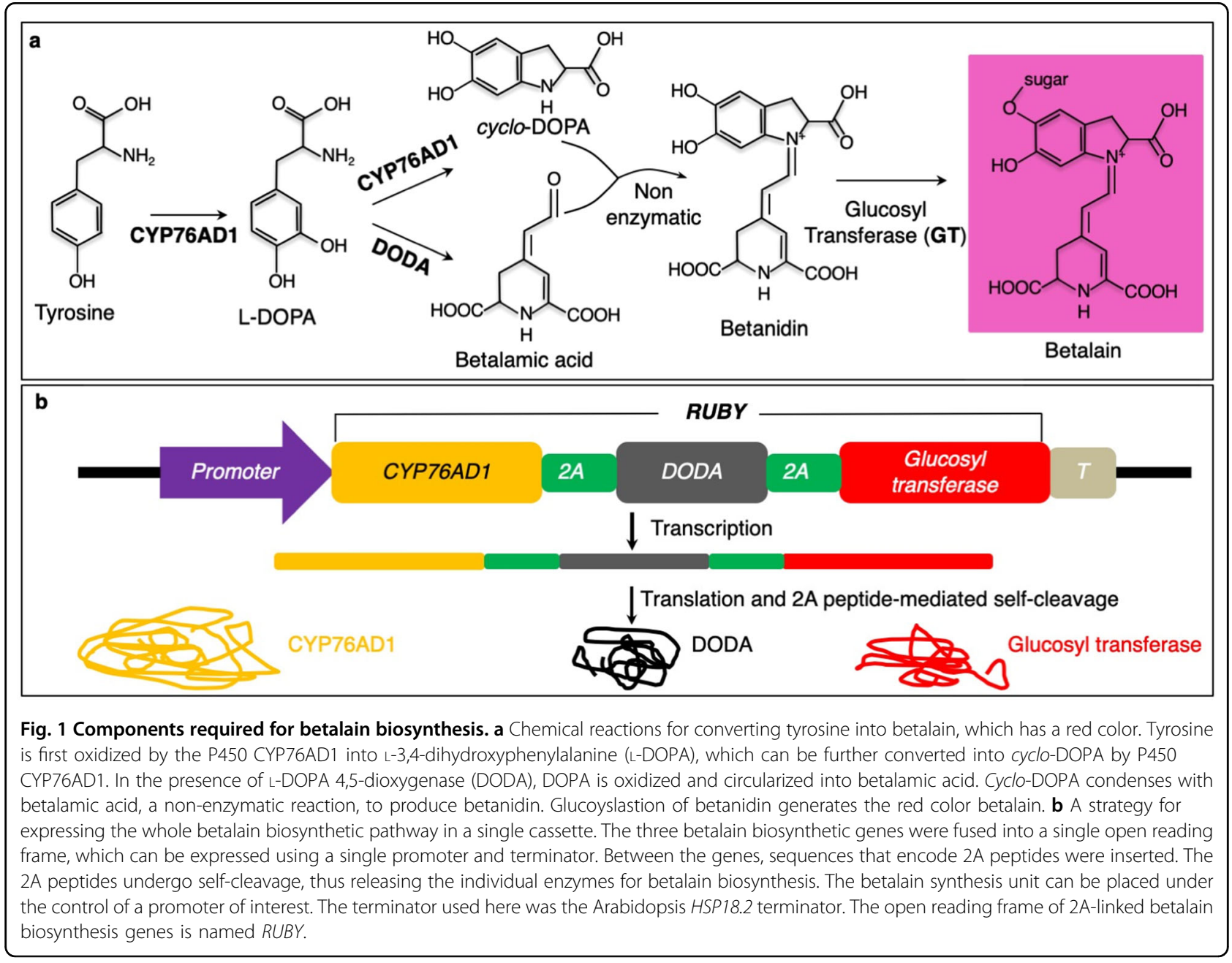




\section{Results and discussion}

\section{Construction of a single open reading frame for the betalain biosynthetic pathway}

Heterologous expression of CYP76AD1, DODA in tobacco, and other plants demonstrated that the betalain biosynthetic pathway can be re-constituted in plant cells ${ }^{13,14}$. In order to use betalain as a visual reporter, we need to effectively co-express the entire pathway using a single promoter. We organized $C Y P 76 A D 1, D O D A$, and Glucosyltransferase into a single open reading frame (Fig. 1b) (Supplemental Fig. 1). The stop codons of $C Y P 76 A D 1$ and $D O D A$ were removed. The three genes were linked by sequences that encode $2 \mathrm{~A}$ peptides (Fig. 1b) (Supplemental Fig. 1) ${ }^{15,16}$. Upon transcription, the single transcript, which includes the coding regions of the three enzymes, produced the three separate enzymes through either 2A-mediated self-cleavage or ribosomal "skipping" (Fig. 1b) ${ }^{17}$. The $2 \mathrm{~A}$ system enables the expression of multiple proteins under the control of a single promoter. We name the 2A-linked unit of CYP76AD1, DODA, and Glucosyltransferase RUBY (Fig. 1b). $R U B Y$ can be expressed when a promoter is placed in front of it. The expression pattern and level of a particular gene may be inferred from the red color of betalain if the gene's promoter is used to drive $R U B Y$ expression.

\section{$R U B Y$ is capable of synthesizing betalain in tobacco}

We first placed $R U B Y$ under the control of Cauliflower Mosaic Virus (CaMV) $35 \mathrm{~S}$ promoter, which is a widely used constitutively strong promoter ${ }^{18}$. To test whether $R U B Y$ can produce functional enzymes for betalain synthesis, we infiltrated tobacco leaves with Agrobacteria that contain $R U B Y$-expressing plasmid (Supplemental Fig. 2 ). Transient expression of $R U B Y$ led to the production of betalain in tobacco leaves, suggesting that the synthetic open reading frame $R U B Y$ can produce the functional enzymes for the synthesis of betalain. Moreover, we observed that betalain was not transported from the spots of Agrobacterium-infiltration spots to other leaves of the plant (Supplemental Fig. 2).

\section{Synthesis of betalain by RUBY in Arabidopsis}

We transformed the 35S:RUBY construct into Arabidopsis using Agrobacterium-mediated floral dipping ${ }^{19}$. Two days after floral dipping, we noticed that the transformed plants displayed patches of red color (Supplemental Fig. 3a), indicating that the $R U B Y$ cassette was functionally expressed and that $R U B Y$ may be used to monitor transient Arabidopsis transformation. Once the seeds from the Agrobacterium-dipped plants were harvested, transgenic seeds could be easily differentiated from non-transgenic seeds (Supplemental Fig. 3b). The transformed seeds had a dark red color (Supplemental Fig. $3 \mathrm{~b})$, demonstrating that $R U B Y$ can be used as a visual selection marker for transgenic events in Arabidopsis. We previously used mCherry as a very effective marker to select transgenic events (Gao et al., 2016), which requires a dissecting microscope with fluoresence capability. RUBY is a better option because it does not require special equipment.

The 35S:RUBY plants produced sufficient amount of betalain to become visually evident (Fig. 2a). Consistent with previous reports that $C a M V 35 S$ promoter is constitutively active, we observed red color in all tissues throughout the plant life cycle (Fig. 2a) (Supplemental Fig. 3c, d). We also expressed $R U B Y$ reporter under the control of the Maize UBIQUITIN promoter, which has been widely used to overexpress genes in monocots ${ }^{20}$. Similar to $35 S: R U B Y$ plants, $U B Q: R U B Y$ plants were also visibly red in leaves, stem, and flowers (Fig. 2b). These results clearly demonstrated that $R U B Y$ could be expressed in Arabidopsis and that our RUBY reporter was able to functionally re-constitute the betalain biosynthetic pathway.

We expressed $R U B Y$ using the seed specific At2S3 promoter, which we previously used to drive mCherry expression in Arabidopsis to facilitate the selection of transgenes ${ }^{6}$. As shown in Fig. 2c, the transgenic plants were indistinguishable from wild type plants. When we checked the seeds in a silique from an At2S3:RUBY T1 plant, $R U B Y$-expressing seeds displayed strong red color, whereas the non-transgenic seeds were green (Fig. 2c). $R U B Y$ can be conveniently used to select single T-DNA insertion events by analyzing the ratio of red seeds to green seeds, which should be $\sim 3: 1$ for single insertions. The At2S3:RUBY results demonstrate that $R U B Y$ could be an effective marker for Arabidopsis transformation. Furthermore, betalain was not widely transported from the sites of synthesis to other tissues as we did not see any red color in leaves (Fig. 2c). We also expressed RUBY under the control of the Arabidopsis YUC4 promoter (Fig. 2d). $Y U C 4$, which encodes a key enzyme in auxin biosynthesis, was shown to express in small regions of embryos, leaves, and flowers ${ }^{21,22}$. GUS signals were observed in leaf tips and apical region of a gynoecium in YUC4 promoter:GUS transgenic plants. We observed similar patterns of betalain production in YUC4:RUBY lines (Fig. 2d).

\section{$R U B Y$ synthesizes betalain in rice}

Unlike Arabidopsis, rice and many other plants are transformed through tissue culture and the formation of calli, which are often mosaic. A visible marker for transformation at tissue culture stage will be very useful. We placed $R U B Y$ under the control of DR5, an auxinresponsive synthetic promoter ${ }^{4}$, which was used to monitor stem cell initiation during tissue culture. We also used the rice ACTIN1 promoter, which is considered a strong promoter, to control $R U B Y$ expression. 


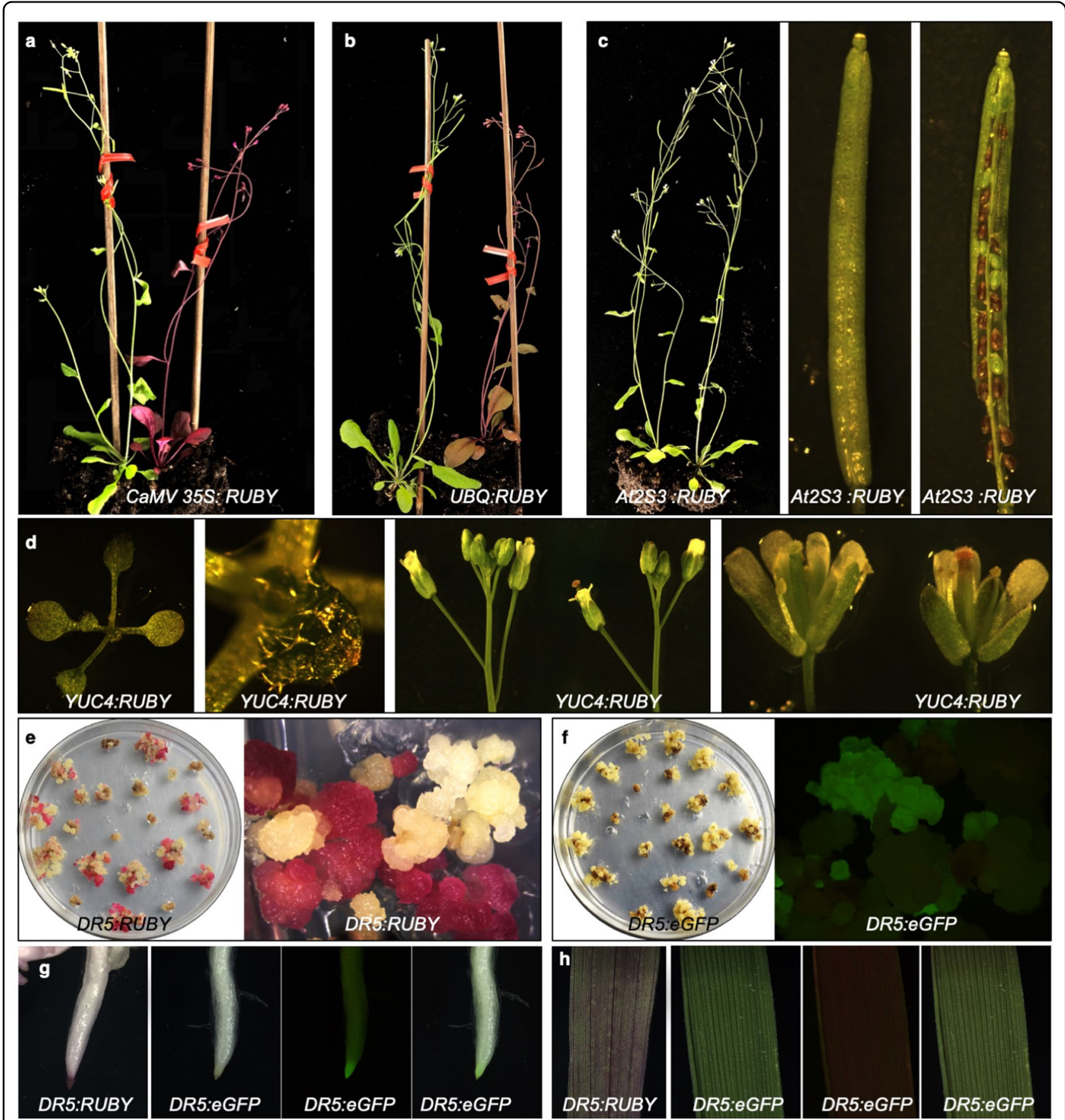

Fig. 2 RUBY serves as an effective reporter for gene expression and plant transformation. a $R U B Y$ expression driven by the CaMV $35 S$ promoter led to a red Arabidopsis plant (right) compared with the WT plant (left). b UBIQUITIN promoter was effective in driving RUBY expression throughout the plant (right). Non-transgenic WT was shown left. c The seed specific promoter At2S3 did not lead to RUBY expression in leaves and stems. The transgenic plant (right) and non-transgenic plant (left) were indistinguishable. The siliques of At2S3:RUBY were similar to those of WT, however, when the silique was opened, the seeds of At2S3:RUBY were clearly red. Moreover, it was obvious that transgenic and no-transgenic seeds were segregating in a silique from a T1 At2S3:RUBY plant. d YUC4:RUBY plants displayed patches of red at the tip of leaves and apical region of a gynoecium. e DR5:RUBY was expressed in rice calli. The red color can be used to distinguish transgenic (red) and non-transgenic calli (white). DR5:eGFP has been used in rice calli $\mathbf{f}$, but it was much more difficult to distinguish transgenic from non-transgenic using DR5:eGFP compared with RUBY. $\mathbf{g}$ Roots of DR5:RUBY and DR5eGFP rice plants, which had similar patterns. The three DR5:eGFP pictures were generated with the same root: bright field (left), $488 \mathrm{~nm}$

fluorescence field (middle), and the merged (right). $\mathbf{h}$ Activation of DR5 promoter in DR5:RUBY leaves was easy to observe whereas DR5:eGFP was much more difficult to detect: bright field (left), $488 \mathrm{~nm}$ fluorescence field (middle), and the merged (right). 
We transformed the DR5:RUBY (Fig. 2e) and OsACTIN1: $R U B Y$ (Supplemental Fig. 4) into rice calli. $R U B Y$ expression rendered calli vividly red. The presence of the $R U B Y$ gene corelated to the observed $R U B Y$ expression in rice calli (Supplemental Fig. 4). We also observed a positive correlation between the brightness of the calli the expression levels of RUBY gene (Supplemental Fig. 4). Introduction of $R U B Y$ made it easier to distinguish the transformed calli from untransformed calli (Fig. 2e). RUBY enables selection of calli that have better expression of transgenes among the hygromycin-resistant calli in the same tissue block (Supplemental Fig. 4). In comparison, DR5:eGFP has been used to visualize transgenic calli (Fig. 2f). Our RUBY system is clearer and much more convenient to operate during tissue culture condition. The DR5:RUBY and DR5:eGFP in rice roots showed similar patterns (Fig. 2g), but DR5:RUBY was easier to observe without any treatments or a change in light conditions. The advantages of DR5:RUBY over DR5:eGFP was also obvious in rice leaves (Fig. 2h). Moreover, $R U B Y$ as a visible marker was very useful for monitoring transgenes in intact adult rice plants (Supplemental Fig. 5).

We demonstrate that our synthetic cassette of betalain biosynthetic genes was able to produce betalain in Arabidopsis and in rice, providing a visible color for monitoring gene expression and plant transformation. We believe that $R U B Y$ will be very useful in large plants such as fruit trees and in field conditions. Because RUBY does not require either special equipment or expensive substrates, $R U B Y$ provides a cost-effective reporter and $R U B Y$ is a convenient alternative to the existing reporters. We envision that $R U B Y$ can be adapted for applications in some microbes and animals because the substrate tyrosine exists in all cells. For example, $R U B Y$ may provide a more convenient marker than $\beta$-galactosidase (LacZ) in yeast two-hybrid screens. Betalain is a natural product and was shown to have health benefits. Using $R U B Y$ as a reporter has less environmental and health concerns compared with antibiotic and/or herbicide resistance markers.

\section{Materials and methods}

\section{Arabidopsis constructs and transformation}

The backbone of the RUBY constructs for Arabidopsis is the plasmid $p H D E$, which was described previously ${ }^{6}$. We took advantage of the P2A peptide, which has the sequence of (GSGATNFSLLKQAGDVEENPGP), to link the CYP76AD1,DODA, and glucosyltransferase (GT) coding regions. The transcriptional terminator used was the HSP18.2 terminator from Arabidopsis. The 2A-linked $C Y P 76 A D 1, D O D A$, and $G T$ unit was named RUBY, which was cloned into $p H D E$ along with the HSP terminator by Gibson assembly at the $X b a \mathrm{I}^{23}$. The entire sequence of $R U B Y$ and terminator was shown in Supplemental Fig. 1.
Various promoters can be cloned into the PmeI site of $p H D E-R U B Y$ to drive $R U B Y$ expression. Promoters were amplified by PCR and the primers used in this study were listed in Supplemental Table 1. We expressed $R U B Y$ using CaMV 35S, Maize UBIQUITIN promoter, At2S3 promoter, and YUC4 promoter to test whether $R U B Y$ can serve as an effective reporter. The constructs were transformed into Arabidopsis Columbia plants by Agrobacterium-mediated floral dipping ${ }^{19}$. Transgenic seeds for 35S:RUBY, UBQ:RUBY, and At2S3: RUBY were easily identified by red color. Transgenic plants for YUC4:RUBY were selected on MS medium containing hygromycin $(16.7 \mu \mathrm{g} / \mathrm{ml})$.

\section{Rice constructs and transformation}

The auxin-responsive promoter (DR5) was composed of a synthetic promoter with 16 repeats of core auxin response element (AuxRE) sequence (TGTCTC) linked with CaMV minimal $35 S$ promoter ${ }^{4}$. The DR5 promoter was synthesized and then cloned into $p D X 2181^{24}$ between BamH I and Pst I sites by Gibson assembly with primer pair Oligo15 and Oligo16 (Supplemental Table 1), resulting in the plasmid $p D R 5: e G F P$.

To construct the $p D R 5: R U B Y$ plasmid, we linked the three betalain biosynthetic genes through $2 \mathrm{~A}$ peptides. Here, we used the F2A peptide, which has the following peptide sequence: QLLNFDLLKLAGDVESNPGP. The RUBY cassette replaced the eGFP in the $p D R 5$ :eGFP plasmid, resulting in the $p D R 5: R U B Y$. The rice version of $R U B Y$ was also detailed in the Supplemental Fig. 1. Both the DR5:RUBY and DR5:eGFP plasmids were transformed into Xiaowei ${ }^{\mathrm{NIP} 25}$ through Agrobacterium-mediated plant transformation following a protocol that was previously described $^{26}$.

\section{Quantitative analysis the expression level of $R U B Y$}

The relative expression levels of $R U B Y$ in the transgenic calli of OsACTIN1: RUBY were determined by reverse transcription quantitative $\mathrm{PCR}$ using the primer pair Oligo26/Oligo27. The primer pair Oligo28/ Oligo29 was specific for the rice UBIQUITIN (UBQ) gene, which served as the endogenous reference gene ${ }^{27}$. The primers were listed in Supplemental Table 1.

\section{Acknowledgements}

This work was partially supported by grants from the National Transgenic Science and Technology Program (2019ZX08010-003; 2019ZX08010-001) to Y.H. and H.Z. T.Z. is a TIGS postdoctoral fellow. We thank Mr. Vitor Pinoti for helping the tobacco transformation.

\section{Author details}

${ }^{1}$ State Key Laboratory of Crop Genetics and Germplasm Enhancement, Nanjing Agricultural University, Nanjing 210095, China. ${ }^{2}$ National Key Laboratory of Crop Genetic Improvement and National Center of Plant Gene Research (Wuhan), Huazhong Agricultural University, Wuhan 430070, China. ${ }^{3}$ Section of Cell and Developmental Biology, University of California San Diego, 9500 
Gilman Drive, La Jolla, CA 92093-0116, USA. ${ }^{4}$ Tata Institute for Genetics and Society-UCSD, La Jolla, CA 92093-0335, USA

\section{Author contributions}

Y.H. and Y.Z. conceived the idea. Y.H. and H.S. conducted the rice experiments. T.Z. performed the Arabidopsis experiments. Y.H., T.Z., and Y.Z. wrote the manuscript.

\section{Data availability}

The plasmids will be available at Addgene.

\section{Conflict of interest}

The authors declare that they have no conflict of interest.

Supplementary Information accompanies this paper at (https://doi.org/ 10.1038/s41438-020-00390-1).

Received: 6 May 2020 Accepted: 18 August 2020

Published online: 19 September 2020

\section{References}

1. Chalfie, M., Tu, Y., Euskirchen, G., Ward, W. W. \& Prasher, D. C. Green fluorescent protein as a marker for gene expression. Science 263, 802-805 (1994).

2. Heim, R., Cubitt, A. B. \& Tsien, R. Y. Improved green fluorescence. Nature $\mathbf{3 7 3}$ 663-664 (1995).

3. Jefferson, R. A., Kavanagh, T. A. \& Bevan, M. W. GUS fusions: beta-glucuronidase as a sensitive and versatile gene fusion marker in higher plants. EMBO J. $\mathbf{6}$, 3901-3907 (1987).

4. Sabatini, S. et al. An auxin-dependent distal organizer of pattern and polarity in the Arabidopsis root. Cell 99, 463-472 (1999).

5. Contag, C. H. \& Bachmann, M. H. Advances in in vivo bioluminescence imaging of gene expression. Annu. Rev. Biomed. Eng. 4, 235-260 (2002).

6. Gao, X., Chen, J., Dai, X., Zhang, D. \& Zhao, Y. An effective strategy for reliably isolating heritable and Cas9-free arabidopsis mutants generated by CRISPR Cas9-mediated genome editing. Plant Physiol. 171, 1794-1800 (2016).

7. He, Y. \& Zhao, Y. Technological breakthroughs in generating transgene-free and genetically stable CRISPR-edited plants. aBIOTECH 1, 88-96 (2020).

8. Misyura, M., Colasanti, J. \& Rothstein, S. J. Physiological and genetic analysis of Arabidopsis thaliana anthocyanin biosynthesis mutants under chronic adverse environmental conditions. J. Exp. Bot. 64, 229-240 (2013).

9. Li, P. et al. Regulation of anthocyanin and proanthocyanidin biosynthesis by Medicago truncatula bHLH transcription factor MtTT8. N. Phytol. 210, 905-921 (2016).
10. Strack, D., Vogt, T. \& Schliemann, W. Recent advances in betalain research. Phytochemistry 62, 247-269 (2003).

11. Xu, J.-J., Fang, X., Li, C.-Y., Yang, L. \& Chen, X.-Y. General and specialized tyrosine metabolism pathways in plants. aBIOTECH, https://doi.org/10.1007/s42994019-00006-w (2019).

12. Polturak, G. \& Aharoni, A. Advances and future directions in betalain metabolic engineering. N. Phytol. 224, 1472-1478 (2019).

13. Polturak, G. et al. Elucidation of the first committed step in betalain biosynthesis enables the heterologous engineering of betalain pigments in plants. N. Phytol. 210, 269-283 (2016).

14. Polturak, G. et al. Engineered gray mold resistance, antioxidant capacity, and pigmentation in betalain-producing crops and ornamentals. Proc. Natl Acad. Sci. USA 114, 9062-9067 (2017).

15. Liu, Z. et al. Systematic comparison of 2A peptides for cloning multi-genes in a polycistronic vector. Sci. Rep. 7, 2193 (2017)

16. Wang, J. \& Chen, H. A novel CRISPR/Cas9 system for efficiently generating Cas9-free multiplex mutants in Arabidopsis. aBIOTECH 1, 6-14 (2020).

17. Sharma, P. et al. 2A peptides provide distinct solutions to driving stop-carry on translational recoding. Nucleic Acids Res. 40, 3143-3151 (2012).

18. Benfey, P. N. \& Chua, N. H. The cauliflower mosaic virus 355 promoter: combinatorial regulation of transcription in plants. Science 250, 959-966 (1990).

19. Clough, S. J. \& Bent, A. F. Floral dip: a simplified method for Agrobacteriummediated transformation of Arabidopsis thaliana. Plant J. 16, 735-743 (1998).

20. Cornejo, M. J., Luth, D., Blankenship, K. M., Anderson, O. D. \& Blechl, A. E. Activity of a maize ubiquitin promoter in transgenic rice. Plant Mol. Biol. 23, 567-581 (1993).

21. Cheng, Y., Dai, X. \& Zhao, Y. Auxin biosynthesis by the YUCCA flavin monooxygenases controls the formation of floral organs and vascular tissues in Arabidopsis. Genes Dev. 20, 1790-1799 (2006).

22. Cheng, Y., Dai, X. \& Zhao, Y. Auxin synthesized by the YUCCA flavin monooxygenases is essential for embryogenesis and leaf formation in Arabidopsis. Plant Cell 19, 2430-2439 (2007).

23. Gibson, D. G. et al. Enzymatic assembly of DNA molecules up to several hundred kilobases. Nat. Methods 6, 343-345 (2009).

24. Ye, R., Zhou, F. \& Lin, Y. Two novel positive cis-regulatory elements involved in green tissue-specific promoter activity in rice (Oryza sativa $L$ ssp.). Plant Cell Rep. 31, 1159-1172 (2012).

25. Hu, S. et al. Xiaowei, a new rice germplasm for large-scale indoor research. Mol. Plant 11, 1418-1420 (2018).

26. Hiei, Y., Ohta, S., Komari, T. \& Kumashiro, T. Efficient transformation of rice (Oryza sativa L.) mediated by Agrobacterium and sequence analysis of the boundaries of the T-DNA. Plant J. 6, 271-282 (1994).

27. He, Y. et al. PINOID is required for formation of the stigma and style in rice. Plant Physiol. 180, 926-936 (2019). 